\title{
Leonardo's three-dimensional relations and some identities
}

\author{
Renata Passos Machado Vieira ${ }^{1}$, Milena Carolina \\ dos Santos Mangueira ${ }^{2}$, Francisco Regis Vieira Alves ${ }^{3}$ \\ and Paula Maria Machado Cruz Catarino ${ }^{4}$ \\ ${ }^{1}$ Department of Mathematics, Federal Institute of Education, Science and Techonology \\ of State of Ceara - IFCE, Treze of Maio, Brazil \\ e-mail: re.passosmegmail.com \\ ${ }^{2}$ Department of Mathematics, Federal Institute of Education, Science and Techonology \\ of State of Ceara - IFCE, Treze of Maio, Brazil \\ e-mail: milenacarolina24@gmail.com \\ ${ }^{3}$ Department of Mathematics, Federal Institute of Education, Science and Techonology \\ of State of Ceara - IFCE, Treze of Maio, Brazil \\ e-mail: fregisdifce.edu.br \\ ${ }^{4}$ University of Trás-os-Montes and Alto Douro - UTAD \\ Vila Real, Portugal \\ e-mail: pcatarino23@gmail.com
}

Received: 18 December 2020 Revised: 17 September 2021 Accepted: 19 September 2021

\begin{abstract}
In this work, new results are explored in relation to the Leonardo sequence. With that, a study about this second order recursive sequence, little explored in the mathematical scope, is briefly presented, relating it to the Fibonacci sequence. Thus, its complexification process is carried out, where from its one-dimensional model, imaginary units are inserted, obtaining Leonardo's three-dimensional numbers. In this way, the imaginary units $i$ and $j$ are inserted. Finally, some three-dimensional identities are presented for Leonardo's numbers.
\end{abstract}

Keywords: Two-dimensional relations, Three-dimensional relations, Leonardo sequence, Fibonacci sequence.

2020 Mathematics Subject Classification: 11B37, 11B39. 


\section{Introduction}

Currently, there are few works on the Leonardo sequence, initially presented by Catarino and Borges [3] and, later, some authors presented studies related to this sequence, such as Shannon [6], Alves and Vieira [1] and Vieira, Alves, and Catarino [7]. Alves et al. [2] report that the absence of information on the historical process of Leonardo's sequence is notorious, with the mathematician Leonardo Pisano (1180-1250) being suspected as the creator of this sequence, due to the name is given and for having a great similarity with the Fibonacci sequence.

Thus, the Fibonacci sequence is a second-order recursive linear sequence, called $F_{n}$, and defined by:

$$
F_{n}=F_{n-1}+F_{n-2}, n \geq 2,
$$

where $F_{0}=0$ and $F_{1}=1$ are the initial terms.

Thus, there is the Leonardo sequence, which is a second-order recursive linear sequence, denominated by $L e_{n}$ and defined by:

$$
L e_{n}=L e_{n-1}+L e_{n-2}+1, n \geq 2,
$$

with $L e_{0}=L e_{1}=1$.

From Eq. (2) it is possible to rewrite this recurrence relation, as performed in [3], presenting a new recurrence, being:

$$
L e_{n+1}=2 L e_{n}-L e_{n-2}, n \geq 2 .
$$

Catarino and Borges [3] present properties and theorems around these Leonardo numbers, as well as a relationship between the Leonardo sequence and the Fibonacci sequence, notably:

Proposition 1.1. For every $n \geq 0$, we have:

$$
L e_{n}=2 F_{n+1}-1 .
$$

Proof. The demonstration is carried out using the finite induction principle. For $n=2$, we have:

$$
\begin{aligned}
L e_{n} & =L e_{n-1}+L e_{n-2}+1 \\
L e_{2} & =L e_{1}+L e_{0}+1 \\
L e_{2} & =3 .
\end{aligned}
$$

Assuming that Eq. (3) is valid for some $n=k, k \in \mathbf{N}$, we have:

$$
L e_{k}=L e_{k-1}+L e_{k-2}+1 .
$$

Thus, it is necessary to prove whether the equation is valid for $n=k+1$. So, using the Eqs. (2) and (1), we have:

$$
\begin{aligned}
L e_{k+1} & =L e_{k}+L e_{k-1}+1 \\
& =\left(2 F_{k+1}-1\right)+\left(2 F_{k}-1\right)+1 \\
& =2\left(F_{k+1}-F_{k}\right)-1 \\
& =2 F_{k+2}-1 .
\end{aligned}
$$

And yet, from Eq. (3), we get:

$$
F_{n+1}=\frac{L e_{n}+1}{2}
$$


With that, in the next sections, the two-dimensional and three-dimensional relations of Leonardo's sequence and identities inherent to this sequence in its complex form will be addressed.

\section{Leonardo's two-dimensional relations}

Initially Harman [4] presented the two-dimensional, three-dimensional recurring relationship and identities of Fibonacci numbers, in order to understand relevant aspects about their respective complexification process.

Similar to the study carried out by Harman in [4] and Oliveira, Alves and Paiva [5], it was possible to carry out an evolutionary process of Leonardo's numbers in order to present their two-dimensional recurrent relationship, based on its one-dimensional form, in which, the dimension increase and the insertion of the imaginary unit $i$.

Based on the work of Vieira, Alves and Catarino [7] which depicts the two-dimensional shape of Leonardo's numbers, where for two integers $n, m \in \mathbb{N}$ in the form $L e(n, m)$, we have:

$$
L e(n, m)=\left[L e(n) \cdot \frac{L e(m)+1}{2}+\frac{L e(m)+1}{2}-1\right]+\left(\frac{L e(n)+1}{2}\right)\left(\frac{L e(m-1)+1}{2}\right) i .
$$

Thus, according to the relationship between the two sequences, in which $F(n+1)=\frac{L e(n)+1}{2}$, one can then restructure the two-dimensional relationship contained in the work of Vieira, Alves and Catarino [7], obtaining:

$$
L e(n, m)=(\operatorname{Le}(n) F(m+1)+F(m+1)-1)+F(n+1) F(m) i .
$$

Still performing a small algebraic transformation, as a way to facilitate calculations for the study of three-dimensional relations, we have that:

$$
L e(n, m)=\left[\operatorname{Le}(n) F(m+1)+F(m-1)\left(\frac{F(m-1)+L e(m-1)}{2}\right)\right]+F(n+1) F(m) i .
$$

\section{Leonardo's three-dimensional relations}

Leonardo's two-dimensional numbers support the study of recurrent three-dimensional relations, in which it is explained for a third dimension. Thus, in addition to the imaginary unit $i$, the imaginary unit $j$ is also inserted.

Thus, in this section, some properties inherent to the three-dimensional relations of Leonardo's sequence will be discussed.

Definition 3.1. Leonardo's three-dimensional numbers are defined with the initial values: $\operatorname{Le}(0,0,0)=1=\operatorname{Le}(0), \operatorname{Le}(1,0,0)=1=\operatorname{Le}(1), \operatorname{Le}(0,1,0)=1+i, \operatorname{Le}(0,0,1)=1+j$, $\operatorname{Le}(1,1,1)=1+i+j, \operatorname{Le}(0,1,1)=1+i+j, \operatorname{Le}(1,0,1)=1+j, \operatorname{Le}(1,1,0)=1+i$, where $i^{2}=j^{2}=-1$, forming the numbers in the form Le $(n, m, p)$ satisfying the following three-dimensional recurrence conditions, where $n, m, p \geqslant 0$ :

$$
\left\{\begin{array}{l}
L e(n, m, p)=2 L e(n-1, m, p)-L e(n-3, m, p) \\
L e(n, m, p)=2 L e(n, m-1, p)-L e(n, m-3, p) \\
\operatorname{Le}(n, m, p)=2 \operatorname{Le}(n, m, p-1)-L e(n, m, p-3) .
\end{array}\right.
$$


Lemma 3.2. The following properties are valid for Leonardo's numbers:

(a) $\operatorname{Le}(n, 0,0)=\operatorname{Le}(n)$,

(b) $L e(n, 1,0)=L e(n)+F(n+1) i$,

(c) $L e(n, 0,1)=L e(n)+F(n+1) j$,

(d) $L e(n, 1,1)=L e(n)+F(n+1) i+F(n+1) j$.

Proof. To demonstrate the property (a) $L e(n, 0,0)=L e(n)$, the relationship $\operatorname{Le}(n, m, p)=$ $2 L e(n-1, m, p)-L(n-3, m, p)$ and the initial values initially defined. Thus, for $m=p=0$, that is, $\operatorname{Le}(n, 0,0)=2 L e(n-1,0,0)-L(n-3,0,0)$, varying $n=(0,1,2,3, \ldots)$. You can see that:

$$
\begin{aligned}
\operatorname{Le}(n, 0,0) & =2 \operatorname{Le}(n-1,0,0)-\operatorname{Le}(n-3,0,0): \\
\operatorname{Le}(3,0,0) & =2 \operatorname{Le}(2,0,0)-\operatorname{Le}(0,0,0)=5=\operatorname{Le}(3) ; \\
\operatorname{Le}(4,0,0) & =2 \operatorname{Le}(3,0,0)-\operatorname{Le}(1,0,0)=9=\operatorname{Le}(4) ; \\
\operatorname{Le}(5,0,0) & =2 \operatorname{Le}(4,0,0)-\operatorname{Le}(2,0,0)=15=\operatorname{Le}(5) ; \\
& \vdots \\
\operatorname{Le}(n-3,0,0) & =2 \operatorname{Le}(n-4,0,0)-\operatorname{Le}(n-6,0,0)=\operatorname{Le}(n-3) ; \\
\operatorname{Le}(n-2,0,0) & =2 \operatorname{Le}(n-3,0,0)-\operatorname{Le}(n-5,0,0)=\operatorname{Le}(n-2) ; \\
\operatorname{Le}(n-1,0,0) & =2 \operatorname{Le}(n-2,0,0)-\operatorname{Le}(n-4,0,0)=\operatorname{Le}(n-1) ; \\
\operatorname{Le}(n, 0,0) & =2 \operatorname{Le}(n-1,0,0)-\operatorname{Le}(n-3,0,0) \\
& =2 \operatorname{Le}(n-1)-\operatorname{Le}(n-3)=\operatorname{Le}(n) .
\end{aligned}
$$

The demonstration of Lemma 3.2, properties (b), (c) and (d) is carried out in a manner analogous to that of property (a).

Lemma 3.3. The following properties are valid:

(a) $L e(0, m, 0)=L e(m)+F(m) i$,

(b) $L e(0, m, 1)=L e(m)+F(m) i+F(m+1) j$,

(c) $\operatorname{Le}(1, m, 0)=\operatorname{Le}(m+1)+F(m) i$,

(d) $L e(1, m, 1)=L e(m)+F(m) i+F(m+1) j$.

Proof. (a) In view of the recurrence $L e(n, m, p)=2 L e(n, m-1, p)-L e(n, m-3, p)$, the second principle of induction on $m$ in $n=p=0$. Thus, varying $m=1,2,3, \ldots, k$, it can be seen that:

$$
\begin{aligned}
\operatorname{Le}(0, m, 0) & =2 \operatorname{Le}(0, m-1,0)-\operatorname{Le}(0, m-3,0): \\
\operatorname{Le}(0,3,0) & =2 \operatorname{Le}(0,2,0)-\operatorname{Le}(0,0,0)=5+2 i=\operatorname{Le}(3)+F(3) i ; \\
\operatorname{Le}(0,4,0) & =2 \operatorname{Le}(0,3,0)-\operatorname{Le}(0,1,0)=9+3 i=\operatorname{Le}(4)+F(4) i ; \\
\operatorname{Le}(0,5,0) & =2 \operatorname{Le}(0,4,0)-\operatorname{Le}(0,2,0)=15+5 i=\operatorname{Le}(5)+F(5) i ;
\end{aligned}
$$




$$
\begin{aligned}
L e(0, k-3,0) & =2 L e(0, k-4,0)-L e(0, k-6,0)=L e(k-3)+F(k-3) i ; \\
L e(0, k-2,0) & =2 L e(0, k-3,0)-L e(0, k-5,0)=L e(k-2)+F(k-2) i ; \\
L e(0, k-1,0) & =2 L e(0, k-2,0)-L e(0, k-4,0)=L e(k-1)+F(k-1) i ; \\
L e(0, k, 0) & =2 L e(0, k-1,0)-L e(0, k-3,0) \\
& =2 L e(k-1)+2 F(k-1) i-L e(k-3)-F(k-3) i \\
& =L e(k)+F(k) i .
\end{aligned}
$$

The demonstration of Lemma 3.3, properties (b), (c) and (d) is carried out in a manner analogous to that of property (a).

Lemma 3.4. The following identities are valid:

(a) $L e(0,0, p)=L e(p)+F(p) j$,

(b) $L e(0,1, p)=L e(p)+F(p+1) i+F(p) j$,

(c) $\operatorname{Le}(1,0, p)=L e(p)+F(p) j$,

(d) $\operatorname{Le}(1,1, p)=L e(p)+F(p+1) i+F(p) j$.

Proof. (a) Applying the second principle of recurrent induction $L e(n, m, p)=2 \operatorname{Le}(n, m, p-1)-$ $L e(n, m, p-3)$ on $p$ for $n=m=0$ and varying $p=1,2,3, \ldots, k$, we have:

$$
\begin{aligned}
& \operatorname{Le}(0,0, p)=2 \operatorname{Le}(0,0, p-1)-\operatorname{Le}(0,0, p-3): \\
& L e(0,0,3)=2 L e(0,0,2)-\operatorname{Le}(0,0,0)=5+2 j=L e(3)+F(3) j ; \\
& \operatorname{Le}(0,0,4)=2 \operatorname{Le}(0,0,3)-\operatorname{Le}(0,0,1)=9+3 j=\operatorname{Le}(4)+F(4) j ; \\
& L e(0,0,5)=2 L e(0,0,4)-L e(0,0,2)=15+5 j=L e(5)+F(5) j ; \\
& L e(0,0, k-3)=2 L e(0,0, k-4)-L e(0,0, k-6)=L e(k-3)+F(k-3) j ; \\
& \operatorname{Le}(0,0, k-2)=2 \operatorname{Le}(0,0, k-3)-\operatorname{Le}(0,0, k-5)=\operatorname{Le}(k-2)+F(k-2) j ; \\
& \operatorname{Le}(0,0, k-1)=2 \operatorname{Le}(0,0, k-2)-\operatorname{Le}(0,0, k-4)=\operatorname{Le}(k-1)+F(k-1) j ; \\
& \operatorname{Le}(0,0, k)=2 \operatorname{Le}(0,0, k-1)-\operatorname{Le}(0,0, k-3) \\
& =2 L e(k-1)+2 F(k-1) j-L e(k-3)-F(k-2) j \\
& =L e(k)+F(k) j \text {. }
\end{aligned}
$$

Validating ownership (a) $\operatorname{Le}(0,0, p)=\operatorname{Le}(p)+F(p) j$.

(b) Using the second principle of recurrent induction $\operatorname{Le}(n, m, p)=2 \operatorname{Le}(n, m, p-1)-L e(n, m, p-$ $3)$ on $p$ for $n=0$ and $m=1$ and varying $p=1,2,3, \ldots, k$, we have: 


$$
\begin{aligned}
& \operatorname{Le}(0,1, p)=2 \operatorname{Le}(0,1, p-1)-\operatorname{Le}(0,1, p-3): \\
& L e(0,1,3)=2 L e(0,1,2)-L e(0,1,0)=6+2 i+j=3 F(3)+F(3) i+F(2) j ; \\
& L e(0,1,4)=2 L e(0,1,3)-L e(0,1,1)=9+3 i+2 j=3 F(4)+F(4) i+F(3) j ; \\
& L e(0,1,5)=2 L e(0,1,4)-L e(0,1,2)=15+5 i+3 j=3 F(5)+F(5) i+F(4) j ; \\
& L e(0,1, k-3)=2 \operatorname{Le}(0,1, k-4)-\operatorname{Le}(0,1, k-6)=\operatorname{Le}(k-3)+F(k-2) i+F(k-3) j ; \\
& L e(0,1, k-2)=2 \operatorname{Le}(0,1, k-3)-L e(0,1, k-5)=L e(k-2)+F(k-1) i+F(k-2) j ; \\
& L e(0,1, k-1)=2 L e(0,1, k-2)-L e(0,1, k-4)=L e(k-1)+F(k) i+F(k-1) j ; \\
& \operatorname{Le}(0,1, k)=2 \operatorname{Le}(0,1, k-1)-\operatorname{Le}(0,1, k-3) \\
& =2 L e(k-1)+2 F(k) i+2 F(k-1) j-L e(k-3)-F(k-2) i-F(k-3) j \\
& =L e(k)+F(k+1) i+F(k) j \text {. }
\end{aligned}
$$

Validating ownership (b) $L e(0,1, p)=L e(p)+F(p+1) i+F(p) j$.

(c) Through the second principle of recurrent induction $\operatorname{Le}(n, m, p)=2 \operatorname{Le}(n, m, p-1)-$ $L e(n, m, p-3)$ on $p$ for $n=1$ and $m=0$ and varying $p=1,2,3, \ldots, k$, we have:

$$
\begin{aligned}
& \operatorname{Le}(1,0, p)=2 \operatorname{Le}(1,0, p-1)-\operatorname{Le}(1,0, p-3): \\
& L e(1,0,3)=2 L e(1,0,2)-L e(1,0,0)=5+2 j=L e(3)+F(3) j ; \\
& \operatorname{Le}(1,0,4)=2 \operatorname{Le}(1,0,3)-\operatorname{Le}(1,0,1)=9+3 j=L e(4)+F(4) j ; \\
& L e(1,0,5)=2 L e(1,0,4)-L e(1,0,2)=15+5 j=L e(5)+F(5) j ; \\
& \operatorname{Le}(1,0, k-3)=2 \operatorname{Le}(1,0, k-4)-\operatorname{Le}(1,0, k-6)=\operatorname{Le}(k-3)+F(k-3) j ; \\
& L e(1,0, k-2)=2 \operatorname{Le}(1,0, k-3)-\operatorname{Le}(1,0, k-5)=\operatorname{Le}(k-2)+F(k-2) j ; \\
& L e(1,0, k-1)=2 L e(1,0, k-2)-L e(1,0, k-4)=L e(k-1)+F(k-1) j ; \\
& \begin{aligned}
L e(1,0, k) & =2 L e(1,0, k-1)-L e(1,0, k-3) \\
& =2 L e(k-1)+2 F(k-1) j-L e(k-3)-F(k-3) j \\
& =L e(k)+F(k) j .
\end{aligned}
\end{aligned}
$$

Validating ownership (c) $L e(1,0, p)=L e(p)+F(p) j$.

(d) According to the second principle of recurrent induction $\operatorname{Le}(n, m, p)=2 \operatorname{Le}(n, m, p-1)-$ $L e(n, m, p-3)$ on $p$ for $n=m=1$ and varying $p=1,2,3, \ldots, k$, we have: 


$$
\begin{aligned}
L e(1,1, p) & =2 L e(1,1, p-1)-L e(1,1, p-3): \\
L e(1,1,3) & =2 L e(1,1,2)-L e(1,1,0)=5+3 i+2 j=L e(3)+F(4) i+F(3) j ; \\
L e(1,1,4) & =2 L e(1,1,3)-L e(1,1,1)=9+5 i+3 j=L e(4)+F(5) i+F(4) j ; \\
L e(1,1,5) & =2 L e(1,1,4)-L e(1,1,2)=15+8 i+5 j=L e(5)+F(6) i+F(5) j ; \\
& \vdots \\
L e(1,1, k-3) & =2 L e(1,1, k-4)-L e(1,1, k-6)=L e(k-3)+F(k-2) i+F(k-3) j ; \\
L e(1,1, k-2) & =2 L e(1,1, k-3)-L e(1,1, k-5)=L e(k-2)+F(k-1) i+F(k-2) j ; \\
L e(1,1, k-1) & =2 L e(1,1, k-2)-L e(1,1, k-4)=L e(k-1)+F(k) i+F(k-1) j ; \\
L e(1,1, k) & =2 L e(1,1, k-1)-L e(1,1, k-3) \\
& =2 L e(k-1)+2 F(k) i+2 F(k-1) j-L e(k-3)-F(k-2) i-F(k-3) j \\
& =L e(k)+F(k+1) i+F(k) j .
\end{aligned}
$$

Validating ownership (d) $L e(1,1, p)=L e(p)+F(p+1) i+F(p) j$.

Theorem 3.5. For the three integers, $n, m, p \in \mathbb{N}$, numbers in the form $L e(n, m, p)$ are described by:

$$
\begin{aligned}
L e(n, m, p)= & F(n+1) F(m+1) L(p)+F(n+1) F(m-1)\left(\frac{F(m-1)+L(m-1)}{2}\right)+ \\
& \left(\frac{F(n-1)+L(n-1)}{2}\right) F(n-1)+F(n+1) F(m) F(p+1) i+ \\
& F(n+1) F(m+1) F(p) j .
\end{aligned}
$$

Proof. So for $n=0$ and $m=2$, we have:

$$
\begin{aligned}
& L e(0,2,3)=2 L e(0,2,2)-L e(0,2,0)=11+3 i+4 j=2 L e(3)+1+F(4) i+2 F(3) j ; \\
& L e(0,2,4)=2 \operatorname{Le}(0,2,3)-\operatorname{Le}(0,2,1)=19+5 i+6 j=2 L e(4)+1+F(5) i+2 F(4) j ; \\
& L e(0,2,5)=2 L e(0,2,4)-\operatorname{Le}(0,2,2)=31+8 i+10 j=2 L e(5)+1+F(6) i+2 F(5) j ; \\
& \operatorname{Le}(0,2, p-3)=2 \operatorname{Le}(0,2, p-4)-\operatorname{Le}(0,2, p-6)=2 \operatorname{Le}(p-3)+1+F(p-2) i+2 F(p-3) j ; \\
& \operatorname{Le}(0,2, p-2)=2 \operatorname{Le}(0,2, p-3)-\operatorname{Le}(0,2, p-5)=2 \operatorname{Le}(p-2)+1+F(p-1) i+2 F(p-2) j ; \\
& \operatorname{Le}(0,2, p-1)=2 \operatorname{Le}(0,2, p-2)-\operatorname{Le}(0,2, p-4)=2 \operatorname{Le}(p-1)+1+F(p) i+2 F(p-1) j ; \\
& \begin{aligned}
\operatorname{Le}(0,2, p) & =2 \operatorname{Le}(0,2, p-1)-\operatorname{Le}(0,2, p-3) \\
& =4 L e(p-1)+2+2 F(p) i+4 F(p-1) j \\
& -2 L e(p-3)-1-F(p-2) i-2 F(p-3) j \\
& =2 L e(p)+1+F(p+1) i+2 F(p) j
\end{aligned}
\end{aligned}
$$


In addition, other properties inherent to this process are stimulated, for $m=1,2,3, \ldots, k$, obtaining:

$$
\begin{aligned}
& L e(0,0, p)=F(1) L e(p)+\left(\frac{F(-1)+L e(-1)}{2}\right) F(-1)+F(0) F(p+1) i+F(1) F(p) j ; \\
& L e(0,1, p)=F(2) L e(p)+\left(\frac{F(0)+L e(0)}{2}\right) F(0)+F(1) F(p+1) i+F(2) F(p) j ; \\
& L e(0,2, p)=F(3) L e(p)+\left(\frac{F(1)+L e(1)}{2}\right) F(1)+F(2) F(p+1) i+F(3) F(p) j ; \\
& L e(0,3, p)=F(4) L e(p)+\left(\frac{F(2)+L e(2)}{2}\right) F(2)+F(3) F(p+1) i+F(4) F(p) j ; \\
& L e(0, k-3, p)=2 L e(0, k-4, p)-\operatorname{Le}(0, k-6, p)=F(k-2) \operatorname{Le}(p) \\
& +\left(\frac{F(k-4)+L e(k-4)}{2}\right) F(k-4) \\
& +(F(k-3) F(p+1)) i+(F(k-2) F(p)) j ; \\
& L e(0, k-2, p)=2 L e(0, k-3, p)-L e(0, k-5, p)=F(k-1) \operatorname{Le}(p) \\
& +\left(\frac{F(k-3)+L e(k-3)}{2}\right) F(k-3) \\
& +(F(k-2) F(p+1)) i+(F(k-1) F(p)) j ; \\
& L e(0, k-1, p)=2 L e(0, k-2, p)-L e(0, k-4, p)=F(k) L e(p) \\
& +\left(\frac{F(k-2)+L e(k-2)}{2}\right) F(k-2) \\
& +(F(k-1) F(p+1)) i+(F(k) F(p)) j ; \\
& L e(0, k, p)=2 L e(0, k-1, p)-\operatorname{Le}(0, k-3, p) \\
& =2\left(F(k) L e(p)++\left(\frac{F(k-2)+L e(k-2)}{2}\right) F(k-2)\right) \\
& -\left(F(k-2) L e(p)+\left(\frac{F(k-4)+L e(k-4)}{2}\right) F(k-4)\right) \\
& \left.=F(k+1) L e(p)+\left(\frac{F(k-1)+L e(k-1)}{2}\right) F(k-1)\right) \\
& +(F(k) F(p+1)) i+(F(k+1) F(p)) j .
\end{aligned}
$$

This proves the veracity of the Theorem 3.5 , by applying it to $n=1,2,3, \ldots, k$, in the situation presented below:

$$
\begin{aligned}
L e(0, m, p)=F & (m+1) L e(p)+\left(\frac{F(m-1)+L e(m-1)}{2}\right) F(m-1)+F(m) F(p+1) i \\
& +F(m+1) F(p) j \\
L e(1, m, p)=F & (m+1) L e(p)+\left(\frac{F(m-1)+L e(m-1)}{2}\right) F(m-1)+F(m) F(p+1) i \\
& +F(m+1) F(p) j
\end{aligned}
$$




$$
\begin{array}{rl}
L e(2, m, p)=2 & F(m+1) L e(p)+2\left(\frac{F(m-1)+L e(m-1)}{2}\right) F(m-1)+2 F(m) F(p+1) i \\
& +2 F(m+1) F(p) j \\
L e(n, m, p)=F & (n+1) F(m+1) L e(p)+\left[F(n+1) F(m-1)\left(\frac{F(m-1)+L e(m-1)}{2}\right)\right. \\
& \left.+\left(\frac{F(n-1)+L e(n-1)}{2}\right) F(n-1)\right]+F(n+1) F(m) F(p+1) i \\
& +F(n+1) F(m+1) F(p) j .
\end{array}
$$

\section{Three-dimensional identities for Leonardo's numbers}

From the study in relation to Leonardo's three-dimensional numbers, some identities are presented in their complex form, relating it to the Fibonacci numbers. It is worth noting that, in Vieira, Alves and Catarino [7] it's possible to obtain two-dimensional identities of these numbers.

Identity 4.1. The following identities are valid for Leonardo's numbers:

(a) The sum of the first n numbers $L e(n, m, p)$ with an index greater than zero is described as:

$$
\begin{aligned}
\sum_{l=1}^{n} L e(l, m, p)= & L e(n+1, m, p)-L e(0, m, p)-L e(1, m, p)-n \\
= & (F(n+2)-2)\left[F(m+1) L e(p)+\left(\frac{F(m-1)-L e(m-1)}{2}\right) .\right. \\
& \quad+\left(\frac{F(n)+L e(n)}{2}\right) F(n)-n
\end{aligned}
$$

(b) The sum of the Le( $n, m, p)$ numbers of even and non-zero index can be described by:

$$
\begin{aligned}
\sum_{l=1}^{n} L e(2 l, m, p)= & L e(2 n+1, m, p)-L e(1, m, p)-n \\
= & (F(2 n+2)-1)\left[F(m+1) L e(p)+\left(\frac{F(m-1)-L e(m-1)}{2}\right) .\right. \\
& \quad+\left(\frac{F(2 n)+L e(2 n)}{2}\right) F(2 n)-n
\end{aligned}
$$

(c) The sum of the Le(n, $m, p)$ numbers of odd and nonzero index can be described by:

$$
\begin{aligned}
\sum_{l=1}^{n} L e(2 l+1, m, p)= & L e(2 n+2, m, p)-L e(2, m, p)-n \\
= & (F(2 n+3)-2)\left[F(m+1) L e(p)+\left(\frac{F(m-1)-L e(m-1)}{2}\right) .\right. \\
& \quad+\left(\frac{F(2 n+1)+L e(2 n+1)}{2}\right) F(2 n+1)-n .
\end{aligned}
$$


Proof. To demonstrate the Identity (a), starting from the relation $L e(n+1, m, p)=L e(n, m, p)+$ $L e(n-1, m, p)+1$, it can be rewritten as $L e(n, m, p)=L e(n+1, m, p)-L e(n-1, m, p)-1$. Thus, we have:

$$
\begin{aligned}
\operatorname{Le}(1, m, p) & =\operatorname{Le}(2, m, p)-\operatorname{Le}(0, m, p)-1 \\
\operatorname{Le}(2, m, p) & =\operatorname{Le}(3, m, p)-\operatorname{Le}(1, m, p)-1 \\
\operatorname{Le}(3, m, p) & =\operatorname{Le}(4, m, p)-\operatorname{Le}(2, m, p)-1 \\
\operatorname{Le}(4, m, p) & =\operatorname{Le}(5, m, p)-\operatorname{Le}(3, m, p)-1 \\
& \vdots \\
\operatorname{Le}(n, m, p) & =\operatorname{Le}(n+1, m, p)-\operatorname{Le}(n-1, m, p)-1
\end{aligned}
$$

With that, performing the telescopic sum and Theorem 3.5, we have

$$
\begin{aligned}
\sum_{l=1}^{n} L e(l, m, p)= & L e(n+1, m, p)-L e(0, m, p)-L e(1, m, p)-n \\
= & F(n+2) F(m+1) L e(p)+F(n+2) F(m-1) . \\
& \left(\frac{F(m-1)+L e(m-1)}{2}\right)+\left(\frac{F(n)+L e(n)}{2}\right) F(n) \\
& +F(n+2) F(m) F(p+1) i+F(n+2) F(m+1) F(p) j \\
& -F(m+1) L e(p)-\left(\frac{F(m-1)-L e(m-1)}{2}\right) F(m-1) \\
& -F(m) F(p+1) i-F(m+1) F(p) j \\
& -F(m+1) L e(p)-\left(\frac{F(m-1)-L e(m-1)}{2}\right) F(m-1) \\
& -F(m) F(p+1) i-F(m+1) F(p) j-n \\
= & {[F(m+1) L e(p)](F(n+2)-2)+\left[\left(\frac{F(m-1)-L e(m-1)}{2}\right) .\right.} \\
& F(m-1)](F(n+2)-2) \\
& +[F(m) F(p+1) i](F(n+2)-2)+[F(m+1) F(p) j](F(n+2)-2) \\
& +\left(\frac{F(n)+L e(n)}{2}\right) F(n)-n \\
= & (F(n+2)-2)\left[F(m+1) L e(p)+\left(\frac{F(m-1)-L e(m-1)}{2}\right) F(m-1)\right. \\
& +F(m) F(p+1) i+F(m+1) F(p) j]+\left(\frac{F(n)+L e(n)}{2}\right) F(n)-n
\end{aligned}
$$

The demonstration of Lemmas 4.1 (b) and (c) are carried out in a manner analogous to Theorem 4.1 (a).

\section{Conclusion}

Recursive linear sequences have been studied over several years, with an emphasis on the Fibonacci sequence. However, in this work, there was a contribution to the evolutionary and complex study of the Leonardo sequence, relating it to the Fibonacci sequence. Thus, imaginary 
units were introduced to enable the process of expanding its dimensionality. Thus, starting from the unidimensionality, the behavior of the three-dimensional relations was investigated, still analyzing some mathematical identities.

It should be noted that the sequence studied was introduced by Catarino and Borges [3], studying their respective two-dimensional relationship presented by Vieira, Alves and Catarino [7].

\section{Acknowledgements}

Part of the development of research in Brazil had the financial support of the National Council for Scientific and Technological Development (CNPq) and a Coordination for the Improvement of Higher Education Personnel (CAPES).

The research development part in Portugal is financed by National Funds through the Foundation for Science and Technology. I. P (FCT), under the project UID/CED/00194/2020.

\section{References}

[1] Alves, F. R. V., \& Vieira, R. P. M. (2019). The Newton Fractal's Leonardo Sequence Study with the Google Colab. International Eletronic Journal of Mathematics Education, 15(2), $1-9$.

[2] Alves, F. R. V., Catarino, P. M. M. C., Vieira, R. P. M., \& Mangueira, M. C. S. (2020). Teaching recurrent sequences in Brazil using historical facts and graphical illustrations. Acta Didactica Napocensia, 13(1), 87-104.

[3] Catarino, P., \& Borges, A. (2019). On Leonardo numbers. Acta Mathematica Universitatis Comenianae, 89(1), 75-86.

[4] Harman, C. J. (1981). Complex Fibonacci numbers. The Fibonacci Quarterly, 19(1), 82-86.

[5] Oliveira, R. R., Alves, F. R. V., \& Paiva, R. E. B. (2017). Identidades bi e tridimensionais para os números de Fibonacci na forma complexa. CQD-Revista Eletrônica Paulista de Matemática, Bauru, 11, 91-106.

[6] Shannon, A. G. (2019). A note on generalized Leonardo numbers. Notes on Number Theory and Discrete Mathematics, 25(3), 97-101.

[7] Vieira, R. P. M., Alves, F. R. V., \& Catarino, P. M. M. C. (2019). Relações bidimensionais e identidades de Leonardo. Revista Sergipana de Matemática e Educação Matemática, 4(2), $156-173$. 